Erratum

\title{
Compensated compactness and the Heisenberg group
}

Loukas Grafakos, Richard Rochberg

Math. Ann. 300, 451-461 (1994)

\section{Range characterization of Radon transforms on quaternionic projective spaces}

\section{Tomoyuki Kakehi}

Math. Ann. 300, 543-555 (1994)

The above articles unfortunately contained various errors which were introduced when they were processed for printing. The publisher deeply regrets this mistake. Both articles are republished in their correct form in this issue. 